\title{
Information and Communication Technology Tools for Competitive Intelligence
}

\author{
Dirk Vriens \\ Radboud University of Nijmegen, The Netherlands
}

\section{INTRODUCTION}

Competitive intelligence (CI) can be described as collecting and processing information about the environment for strategic purposes (cf. Kahaner, 1997). To formulate a strategy, an organization needs to collect and process information about its environment-about, for instance, competitors, customers, suppliers, governments, technological trends, or ecological developments. Collecting and processing environmental information has, of course, always been important. However, because of the increasing complexity and dynamics of the environment, the pressure to produce relevant, timely, "actionable" intelligence increases as well. At the same time, the amount of available data about the environment also increases.

To deal with this problem, it is necessary to structure intelligence activities, and many organizations use information and communications technology (ICT) to this end. They use, for instance, different types of Internet or intranet applications, so-called data warehouses, groupware applications, or applications specifically tailored to the organizations' intelligence needs. The number of possible ICT tools for CI is large, and an important question for organizations is which tools they should select and implement for their CI activities. Organizations face difficulties in selecting, implementing, and using these tools. Many ICT tools, for instance, lead to an information overload-to large collections of irrelevant data - or are expensive applications that only marginally contribute to the production of intelligence.

To select proper ICT tools for CI, an organization needs to understand the role of ICT for CI. This paper intends to address this understanding. In particular, it will present a short overview of the available tools and it will discuss a procedure for selecting appropriate ICT tools.

\section{BACKGROUND}

To understand the role of ICT for CI and to select proper ICT tools, the CI process needs to be clarified. To describe this process, several authors (cf. Fuld \& Company, 2002; Gilad \& Gilad, 1988; Herring, 1999; Kahaner, 1997) propose to use the intelligence cycle, consisting of four stages.
1. Direction. In this stage organizations determine their strategic information requirements; that is, they determine about what environmental aspects data should be collected. These aspects are also known as competitive intelligence needs (Fleisher, 2001) or key intelligence topics (Kahaner, 1997).

2. Collection. In this stage, the required data are collected. To this end, one needs to (a) determine what sources are available, and (b) access these sources and retrieve data from them.

3. Analysis. This stage focuses on interpreting data from a strategic point of view to determine their strategic relevance (if a piece of data is strategically relevant, the term intelligence is used; cf. Kahaner, 1997; Vriens, 2004). For this analysis, a model of what is relevant for the organization should be available. Often, tools such as Strengths, Weaknesses, Opportunities and Threats (SWOT) analysis, scenario analysis, war gaming, or competitor profiling are used to arrive at such a model (cf. Cook \& Cook, 2000).

4. Dissemination. Here, the intelligence is made available for strategic decision making. That is, the intelligence should be presented clearly and distributed to relevant decision makers who use it to evaluate current strategic options and to generate, compare, select, and implement new ones.

Using this cycle as a background, many authors acknowledge the possible and actual use of ICT applications for CI activities (see, for instance, Chen, Chau, \& Zeng, 2002; Cook \& Cook, 2000; Fleisher \& Blenkhorn, 2001; Fuld \& Company, 2002). It is also a background for discussing the shortcomings of ICT applications for CI (e.g., Cook \& Cook; Fuld \& Company). In line with these authors, we will use this cycle of intelligence activities as a framework for reviewing, classifying, and selecting ICT applications, as will become apparent in the next sections.

\section{A CLASSIFICATION OF ICT TOOLS FOR CI}

ICT tools for CI can be classified according to two "dimensions": (a) their contribution to one or more stages of the intelligence cycle and (b) the specificity of the tool-that 
is, a tool can be either a general ICT tool used for intelligence activities (like a groupware application, used for direction activities) or a tool specifically tailored to one or more intelligence activities. Below, we first discuss the Internet as a "general" ICT tool for all CI activities. Next, we pay attention to other ICT tools, both general and specific. Finally, we discuss so-called business intelligence applications as a specific set of ICT applications.

\section{The Internet as a Tool for $\mathrm{Cl}$}

CI practitioners rely heavily on the use of the Internet for their intelligence activities. Some authors report the use of the Internet for supporting the direction, analysis, and dissemination stages, for example, Web Enabled Technology (WET) applications enhancing communication and collaboration regarding results of this stages (e.g., Teo \& Choo, 2001). However, most attention has been paid to the use of the Internet for collection activities. Among the reported uses of Internet applications for collection activities are the following.

- $\quad$ Search engines

The CI literature discusses many types of search engines and their application for $\mathrm{CI}$ (cf. Chen et al., 2002; Cook \& Cook, 2000).

- Tools for outsourcing collection activities

A part of the collection activities can be outsourced to some (automated) service or tool offered via the Web. Particularly popular is the use of commercial online databases (Kahaner, 1997; Chen et al., 2002). Another example is the use of Web robots or agents that "automatically traverse the hyperlink structure of the WWW [World Wide Web] to locate and retrieve information" (Tan \& Kumar, 2002, p. 9).

- Tools for text analysis Tools supporting the collection of data in (large) Internet-based text files are used.

- Tools for monitoring changes on the Web

- Tools for collecting data about the electronic behavior of Internet users. These tools work, for instance, by identifying users' navigational patterns (cf. Tan \& Kumar, 2002).

- Internet tools for collaboration in collection activities. Internet applications can be used to facilitate the collaboration in collection networks (networks of people performing collection activities).

\section{General and Specific ICT Tools for CI}

Besides using general Internet applications for CI purposes, other general ICT applications can be used as well.
Examples are tools supporting the following.

- $\quad$ Supporting the process of identifying strategic information needs such as different types of groupware or software supporting group model building (e.g., Vennix, 1996).

- $\quad$ Supporting specific methods used in analysis, for instance, system-dynamics software enabling CI professionals to run simulations with certain data, and thus helps to establish their relevance.

- Supporting (management of and collaboration in) the process of analysis. Specific groupware applications may serve this purpose.

- $\quad$ Supporting the dissemination of intelligence-for example, applications supporting (a) the presentation of the intelligence in a suitable format and/or (b) the transmission of reports throughout the organization

There is also a class of ICT applications specifically designed for one or more CI activities. Fuld \& Company (2002) analyzed and evaluated a number of such dedicated CI software packages. They concluded with the following.

(1) The "CI software cannot drive the CI process" (p. 2), but it can help in collecting data, in reporting and communicating intelligence, and in supporting the work flow and collaboration.

(2) No application can deal with all the intelligence stages adequately.

(3) No application can "truly conduct qualitative analysis" (p. 10), but some tools seem promising in assisting $\mathrm{CI}$ analysts to see novel linkages.

\section{Business Intelligence Applications}

For some time, the terms competitive intelligence and business intelligence (BI) were used as synonyms. Nowadays, however, the term business intelligence is often used to indicate a specific set of ICT tools. These BI tools refer to ICT tools enabling (top) management to produce overviews of and analyze relevant organizational data needed for their (strategic) decision making. BI tools typically include data warehouses and tools for extracting and presenting information from them (cf. Cook \& Cook, 2000; Zanasi, 1998). In essence, a data warehouse is a large database in which data from many different databases (e.g., transactional, financial, or personnel databases) are copied and stored in such a way that they are readily accessible. To access, view, and analyze these data, specific tools are developed (e.g., data-mining tools for finding relations between classes of data). Using data 
warehouses and their associated tools to produce intelligence is not unproblematic. Examples of reported problems are the following (cf. Cook \& Cook; Fuld \& Company, 2002).

1. Most data warehouses only cover internal data (generated in the transactions of the organization; Fuld \& Company, 2002). This is a major drawback when treating BI tools as CI tools. However, once data warehouses incorporate relevant external data, they may become valuable CI tools as well.

2. The focus of BI software is primarily on quantitative analysis while CI relies heavily on qualitative data.

3. Data warehouses cause cost and implementation issues: They require large budgets and much implementation time and effort.

4. Cook and Cook (2000) assert that one cannot expect BI software to produce intelligence. Data resulting from analyzing data in a warehouse should still "be analyzed and directly applied to a specific problem to become intelligence" (p.165).

\section{HOW TO SELECT ICT FOR CI}

To select appropriate ICT tools for CI, given specific organizational CI needs, organizations can use three classes of criteria: (a) criteria regarding the contribution of ICT applications to one or more stages of the intelligence cycle, (b) criteria regarding the CI infrastructure, and (c) criteria pertaining to costs. These three classes correspond to the criteria used in the selection of ICT applications in general (cf. Parker, Benson, \& Trainor, 1988).

\section{Criteria Regarding the Contribution to One or More Stages of the Intelligence Cycle}

A (candidate) ICT application should contribute to one or more stages of the intelligence cycle. Criteria to evaluate the contribution of an ICT application for one or more stages may refer to its appropriateness to deliver the desired products for the stage, and to whether it fits the process leading to these products (cf. Fuld \& Company, 2002). For example, collection applications should be evaluated regarding their appropriateness to collect the desired data (e.g., patent data). Applications should also match process aspects. For example, if particular methods are used to analyze data, such as a SWOT analysis, candidate applications can be evaluated regarding their appropriateness in supporting these particular methods.

\section{Criteria Regarding the Relation with the $\mathrm{Cl}$ Infrastructure}

The CI infrastructure consists of three parts: (a) the technology supporting the CI activities (ICT hardware, software, and telecommunications technology), (b) the human resources needed to carry out the CI activities, and (c) the organizational structure of these activities, that is, how CI tasks and responsibilities are divided and allocated. Each possible ICT application should match all three parts-from these three parts, specific criteria can be derived.

The criteria regarding the technology part of the infrastructure focus on the question of whether the candidate application fits the current technology. This fit depends on the current set of ICT applications used to support the CI activities. Does the application fit into this set? Does it deliver more functionalities than this set? Is an easy link between the applications in this set (if desirable) possible? Other questions for judging the fit to the technology have to do with the implementation and maintenance of the application: Does the current hard- and software permit the implementation of the application, or does it require large changes? Is the application reliable? Yet another question for judging the fit to the technology has to do with the contribution of an application to the improvement of the infrastructure; for example, through a particular application, obsolete applications are replaced (or may be replaced more easily), or through a particular application, other stateof-the-art applications can be implemented and used more easily. A data warehouse, for instance, can be seen as a contribution to the current infrastructure because it enables all kinds of tools for visualizing and analyzing (internal) data.

The second set of criteria reflects the fit of the application to the human resources, that is, whether it fits the existing skills, knowledge, and attitudes of those who are carrying out $\mathrm{CI}$ activities. Important questions are, for instance, whether the required knowledge and skills are acquired easily, or whether the CI staff is motivated to integrate the ICT application into their daily routines.

The third set of infrastructural criteria reflects the fit of the application to the current definition and allocation of CI tasks and responsibilities (see Gilad \& Gilad, 1988, for several ways of defining and allocating them). It does not make sense to install groupware for the direction stage if direction is not seen as a group process. The same holds for using ICT tools structuring the collection and analysis activities if the whole CI process has a highly informal nature. In some cases, the CI task structure may be designed poorly and ICT can act as a leverage 
to change it. An ICT tool can be valued because of its contribution to the improvement of the task structure. For instance, a groupware application may be valued for its contribution to structure the direction stage as a group process.

\section{Criteria Pertaining to Costs}

These criteria refer to the costs of the application itself, its implementation (e.g., project costs, training, etc.), and its maintenance. These costs may be calculated by different methods (cf. Parker et al., 1988).

\section{Selecting ICT for CI Using the Three Types of Criteria}

To evaluate the appropriateness of an application for CI in a particular organization, the application should be "scored" regarding all three classes of criteria. To this end, the individual criterion in a class should each be identified, valued, and integrated into an overall score for the class. We will not treat all these substeps in this section; rather, we will indicate how these overall scores can be used to select of ICT for CI.

The overall scores of each class express (a) the contribution of a particular application to one or more of the intelligence stages, (b) the fit of the contribution to the CI infrastructure, and (c) the costs related to an application. These scores can be plotted in a graph (see Figure 1).

In this figure, the scores of several applications are depicted (the size of the circles reflects the costs related to the application). For instance, Application 1 may be a data warehouse (with an internal focus). This application

Figure 1. Classification of ICT applications for CI using three dimensions (see text)

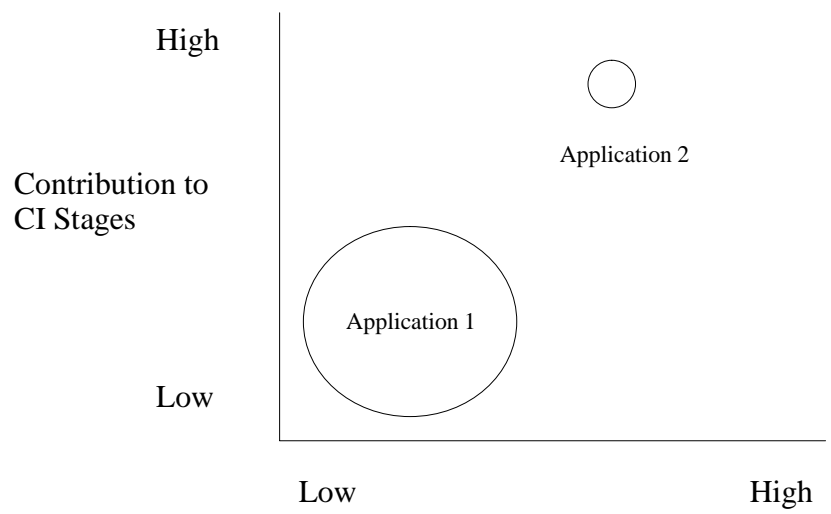

Fit / Contribution to CI Infrastructure may be very expensive, contributes only partly to the intelligence stages (it contributes to the collection stage, but due to its internal focus, its contribution to the CI stages is low). The data warehouse may contribute to the general infrastructure, but, in our view, contributes only partly to the CI infrastructure. In contrast, a data warehouse with explicit external linkages would still be very expensive, but may score higher on both other dimensions. Application 2 may be a groupware application. These applications are moderately expensive, may contribute to the direction stage, and fit the infrastructure in several ways.

An organization may treat several ICT tools to support their CI activities in this way and eventually select some.

\section{FUTURE TRENDS}

Many organizations recognize the importance of supporting CI activities by means of ICT. Although many ICT applications are already available, the possibilities of using ICT for CI will increase rapidly. Some of the trends that may be acknowledged are the following.

- A convergence of BI and CI applications (e.g., data warehouses and associated software also tied to external and qualitative data; cf. Li, 1999)

- $\quad$ Using ICT for qualitative data may increase (e.g., Chen et al. 2002)

- Using the Internet for more than just collection activities (e.g., for collaboration and dissemination purposes; cf. Teo \& Choo, 2001)

- Improvement of Internet applications for collection (More efficient and effective collection applications will continue to emerge.)

- Implementing CI applications can be seen as a process by means of which the CI process and infrastructure can be reanalyzed.

- Improvement of analysis applications (cf. Fuld \& Company, 2002)

\section{CONCLUSION}

To select and use proper ICT tools for supporting the CI process, organizations should know (a) what the CI process is, (b) what the role of ICT (tools) in this process can be, and (c) how to evaluate the role of ICT (tools) for their own CI process. In this chapter, we discussed the role of ICT tools in the CI process. We classified several types of ICT tools relevant for supporting CI activities: the Internet, general and specific applications for supporting CI activities, and business intelligence applications. In 
the last part of this chapter, we discussed three classes of criteria organizations can use in evaluating and selecting ICT tools for their CI processes.

The possibilities of ICT to support CI activities will probably only increase. To make full use of its potential and to avoid many of the pitfalls associated with using ICT for CI, organizations should be careful in selecting and implementing ICT applications for CI purposes. They should know the possibilities of ICT to deliver internal and external data and its capacity for supporting CI activities, and they should treat ICT as a part of the whole infrastructure.

\section{REFERENCES}

Chen, H., Chau, M., \& Zeng, D. (2002). CI-spider: A tool for competitive intelligence on the Web. Decision Support Systems, 34, 1-17.

Cook, M., \& Cook, C. (2000). Competitive intelligence. London: Kogan Page.

Fleisher, C. G. (2001). An introduction to the management and practice of competitive intelligence (CI). In C. G. Fleisher \& D. L. Blenkhorn (Eds.), Managing frontiers in competitive intelligence (pp. 3-18). Westport, CT: Quorum.

Fleisher, C. G., \& Blenkhorn, D. L. (Eds.). (2001). Managing frontiers in competitive intelligence. Westport, CT: Quorum.

Fuld \& Company. (2002). Intelligence software report 2002. Retrieved March 2003 from http://www.fuld.com

Gilad, B., \& Gilad, T. (1988). The business intelligence system. New York: Amacon.

Herring, J. P. (1999). Key intelligence topics: A process to identify and define intelligence needs. Competitive Intelligence Review, 10(2), 4-14.

Kahaner, L. (1997). Competitive intelligence. New York: Touchstone.

Li, C. (1999, Summer). ERP packages: What's next? Information Systems Management, 16 (3), 31-35.

Mahony, T. M. (1998). Data warehousing and CI: An evaluation. Competitive Intelligence Review, 9(1), 38-43.

Parker, M. M., Benson, R. J., \& Trainor, H. E. (1988). Information economics: Linking business performance to information technology. Englewood Cliffs, NJ: Prentice Hall.
Tan, P., \& Kumar, V. (2002). Discovery of Web robot sessions based on their navigational patterns. Data Mining and Knowledge Discovery, 6, 9-35.

Teo, T. S. H., \& Choo, W. Y. (2001). Assessing the impact of using the Internet for competitive intelligence. Information \& Management, 39, 67-83.

Vennix, J. A. M. (1996). Group model building. Chichester, England: Wiley.

Vriens, D. (Ed.). (2004). Information and communication technology for competitive intelligence. Hershey, PA: IRM Press.

Vriens, D., \& Philips, E. A. (1999). Business intelligence als informatievoorziening voor de strategievorming. In E. A. Philips \& D. Vriens (Eds.), Business intelligence, (pp.11-44). Deventer, Netherlands: Kluwer.

Zanasi, A. (1998). Competitive intelligence through data mining public sources. Competitive Intelligence Review, $9(2), 44-54$.

\section{KEY TERMS}

Business Intelligence:The term business intelligence is used in two ways: (a) as a synonym for competitive intelligence and (b) to indicate a specific set of ICT tools to support managerial decision making. This set of tools often consists of a data warehouse and the tools to store, retrieve, and present the information it contains (e.g., data-mining software).

Competitive Intelligence: In the literature, two definitions are used: a product definition and a process definition. In the product definition, competitive intelligence is defined as information about the environment, relevant for strategic purposes. The process definition highlights producing and processing this environmental information. Process definitions often refer to the intelligence cycle.

ICT (Information and communication technology): Can be used to indicate the organization's technological infrastructure (comprising of all hardware, software, and telecommunications technology) and to indicate one or more specific collections of hardware, software, and telecommunications technology (i.e., one or more ICT applications).

ICT Selection: The process of selecting proper parts of the technological infrastructure and/or specific applications. In the context of CI, specific criteria are used: criteria regarding costs, appropriateness in supporting 
intelligence activities, and their fit or contribution to the CI infrastructure.

Intelligence Cycle: This is a cycle of four stages (collections of intelligence activities). The stages are direction (determining the strategic [external] information requirements), collection (determining, locating, accessing, and retrieving sources to obtain data about the environment), analysis (determining the strategic significance of the environmental data), and dissemination (of the intelligence to strategic decision makers).

Intelligence Infrastructure (CI Infrastructure): The intelligence infrastructure comprises all technological, human-resources, and organizational means needed to manage, support, and perform the intelligence activities. It consists of three subinfrastructures: the technological, the human-resources infrastructure, and the organizational infrastructure. 